\title{
SALICE Project: Satellite-Assisted Localization and Communication Systems for Emergency Services
}

\author{
E. Del Re, S. Jayousi, S. Morosi, L. S. Ronga \\ University of Florence
}

M. De Sanctis, E. Cianca, M. Ruggieri

University of Rome Tor Vergata

E. Falletti

Istituto Superiore Mario Boella

A. Iera, G. Araniti

University Mediterranea of Reggio Calabria

C. Sacchi

University of Trento

\section{INTRODUCTION: SALICE PRDJECT MOTIVATION}

Future emergency systems will be characterized by the integration of meshed heterogeneous networks based on both satellite and terrestrial segments [1], [2]. In this architecture the satellite infrastructure will play a lead role because of its independence from the catastrophic event as well as its ability to deliver information over a large area, provide localization information, and collect data from the sensors deployed on the territory. Particularly, the case of natural or man-originated disasters raises the urgent need for immediate restoration of a minimal level of connectivity in order to provide first response to emergency requests, coordinate rescue teams, and allow survivors to communicate with the external world [3]. On the other hand, the cooperation among communications and navigation systems, components, and services will be key features of future emergency systems. The integration of navigation and communication systems allows the exploitation of both navigation information for communication purposes (optimization of communication techniques, interference reduction, and location-based information services delivery) and communication support for navigation purposes (high precision localization and cooperative positioning) [4], [5]. The main goal of the Satellite-Assisted Locallzation and Communication Systems for Emergency Services (SALICE)

Corresponding author's address: M. De Sanctis, University of Roma, Electronics Engineering, Via del Politecnico, 1, Roma, 0013, Italy. E-mail: de.sanctis@ing.uniroma2.it. Manuscript SYSAES-2012-0044r received February 27, 2012, revised September 3, 2012, and ready for publication January 29, 2012.

Review handled by M. Greco.

0885/8985/13/ \$26.00 @ 2013 IEEE project was the investigation of the open issues related to this scenario, the proposal of a proper system architecture, and the establishment of a definition of the innovative solutions that have proven beneficial for emergency operators.

The project focused on two main aspects: achieving global coverage of the emergency area and developing a reconfigurable cooperative NAV/COM system. As for the global coverage objective, proper cooperative schemes, which are based on the use of gap fillers and repeaters in digital video broadcasting - satellite to handheld (DVB-SH) systems, have been defined and analyzed. These solutions have proven to be effective in providing quality of service (QoS) for the considered communications and are characterized by an affordable complexity, which is suitable for the deployment in the scenarios we investigated. In fact, their features make their adoption viable, particularly in the intervention phases, immediately after the disaster. Reconfigurability, interconnection, and interoperability aspects were analyzed and proper strategies of cooperation between high altitude platforms (HAPs) and ad hoc deployed networks, e.g., MANETs (mobile ad hoc networks), were studied. These solutions can support search and rescue units by providing remote connectivity to emergency control centers (ECCs) and supporting local connectivity, which is guaranteed by ad hoc deployed wireless networks. These wireless networks also can compensate for any lack of direct satellite visibility in hostile propagation environments.

In regard to the objective of developing a reconfigurable cooperative NAV/COM system, in the framework of the considered architecture, an original assisted localization technique has been proposed. It is based on the integration of GPS information and the information received from other in loco terminals. Addressing one of the main operational scenarios, the proposed technique permits the localization of partially obscured Global Navigation Satellite System 


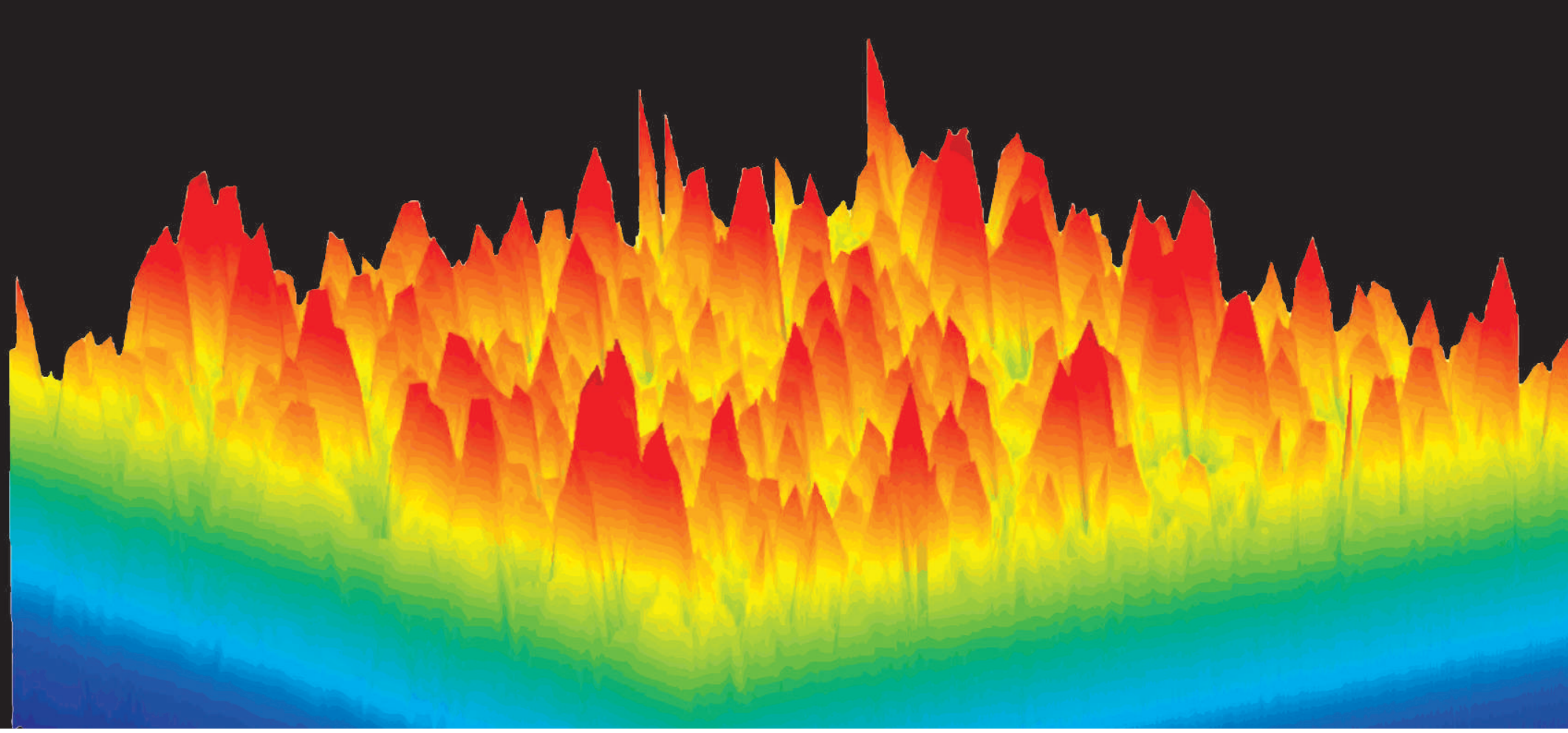

(GNSS) terminals thanks to the transmission of aiding information coming from GNSS line-of-sight terminals. This algorithm has been studied by simulations and implemented by software-defined radio (SDR) technology, enabling the development of a software device (which is defined as the SALICE terminal) to be adopted in the emergency context. The achieved results have shown that this technique can be considered for commercial applications.

\section{SALICE PROJECT OVERVIEW}

The SALICE project was a two-year (October 2008-September 2010) Italian National Research Project, which was funded by the Italian Ministry of University and Research (MIUR) in the framework of PRIN 2007 (Research Project of Relevant National Interest). The project partners were University of Florence (coordinator), Polytechnic of Turin, University of Trento, Mediterranean University of Reggio Calabria, and University of Rome Tor Vergata.

As previewed in the Introduction, the SALICE project's goals were to identify the system architecture and the solutions to be adopted in future integrated reconfigurable NAV / COM systems, as well as to evaluate their feasibility in realistic emergency scenarios. The main pursued objectives are: the integration of self-organizing space and terrestrial segments in a single network infrastructure; the implementation of a reconfigurable NAV/COM SDR-based terminal; the optimization of cooperative localization algorithms and resources management strategies to be adopted in the emergency areas.

\section{BASELINE SCENARIQ AND SYSTEM ARCHITECTURE}

The identification of both the application scenarios and the architectural specifications came out from a thorough anal- ysis of the feedback of potential end users; this procedure created the definition of the emergency system requirements and, consequently, the design of new solutions to guarantee $\mathrm{NAV} / \mathrm{COM}$ services in the area of intervention.

The SALICE baseline scenario is depicted in figure 1. It is worth noticing that the terms and definitions used to indicate the emergency actors are based on [6], although, sometimes, they are specialized or integrated to better comply with the situations of interest for the SALICE system.

The architecture of the emergency network relies on the design of a very robust, reliable, and flexible NAV/COM network (independent of pre-existing public networks, which could have been damaged by the disaster) to be used

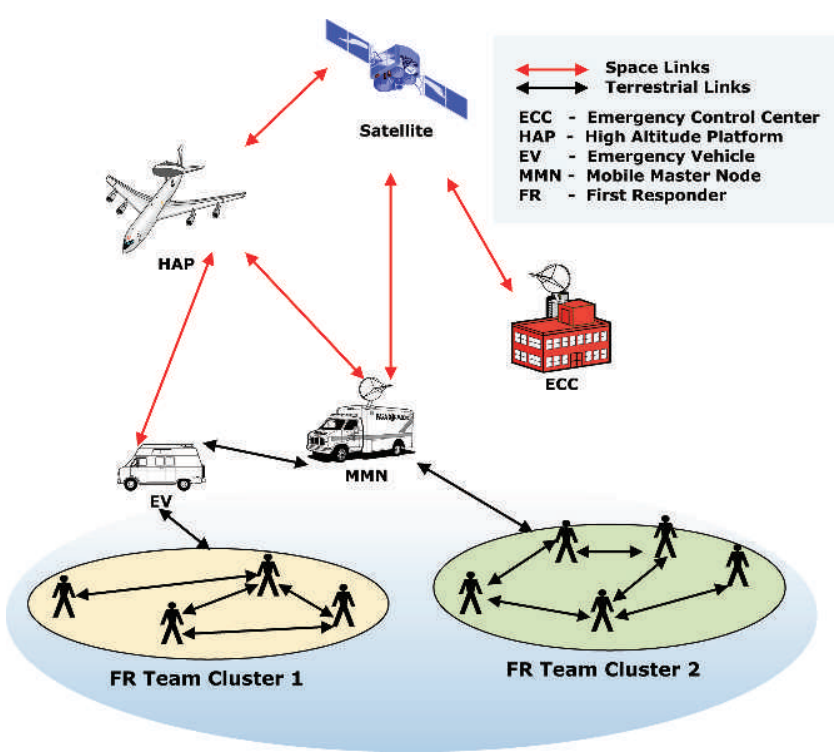

Figure 1.

SALICE baseline scenario. 
for communication among the main rescue teams involved in the emergency operations. A temporary mobile station placed at the perimeter of the emergency area (MMN, mobile master node) can allow the communication between the ECC, the emergency vehicles (EVs), and the first responders (FRs) through a satellite/HAP radio link or through a transportable cellular base station. Both short-range (FR-FR and EV-FR) and long-range (EV/MMN-ECC) radio links are considered together with their integration.

\section{PRDJECT RESEARCH AREAS}

The definition of the baseline scenario and the system architecture provides the guidelines for the investigation of the two main project research areas whose objectives and expected results are summarized in the following:

- Integration of heterogeneous communication systems for global coverage aimed at guaranteeing global coverage of the emergency area, allowing the connection of the incident area with external areas through the integration and cooperation of space (satellite and HAPs) and terrestrial segments, and supporting the provision of NAV/COM services within the area of intervention through the design of an efficient short-range network. Radio resources management strategies, multicast mechanisms, and protocol solutions for location/environment data and information delivery through heterogeneous wireless networks were investigated.

- A reconfigurable and cooperative NAV/COM system aimed at designing and implementing an SDR-based handset with fully reconfigurable software (SALICE terminal), which integrates navigation and communication capabilities and modifies itself to cope with any $\mathrm{NAV} / \mathrm{COM}$ requirements for the emergency operations coordination. A core localization framework (CLF) able to seamlessly cope and self-adapt with heterogeneous and time-varying operational conditions was defined.

\section{SALICE RESULTS}

The most significant results achieved within the two main SALICE project research areas are presented in the following, starting from the problem analysis to the solution development and validation.

\section{ACHIEVING GLOBAL COVERAGE OF THE EMERGENCY AREA}

In an emergency scenario, several radio links with different communication modes and coverage capabilities can coexist; this section deals with the integration of heterogeneous communication systems within the emergency NAV/COM network. This network can be split into two different subnetworks based on their coverage features: a long-range network (LRN) and a short-range network (also called incident area network, IAN). The LRN com- prises Geostationary Earth Orbit (GEO) and Low Earth Orbit (LEO) satellites and HAP systems that enable communication between the incident area and the external zones [7]-[9], while the IAN is a self-forming temporary ad hoc network deployed on the scene of an incident to replace the damaged network infrastructure and is able to support personal and local communications among different public safety end users.

In the following, possible cooperative solutions for both hybrid satellite terrestrial systems and high altitude platforms - mobile ad hoc network (HAP - MANET) networks are defined and studied and the behavior of coexisting air interfaces in the IAN is analyzed.

1) Satellite/terrestrial cooperation: among the different phases of public emergency situation management (preparedness, response, recovery, and mitigation), the adoption of a hybrid satellite terrestrial cooperative system turns out to be one of the most feasible solutions in the first-response phase of a disaster. With the aim of connecting the rescue teams operating in the emergency area with the outside world, the implementation of a hybrid satellite terrestrial cooperative single frequency network (SFN) is analyzed. In particular, the provision of reliable communication in the absence of available terrestrial infrastructure and a good channel propagation condition forces to investigate both the diversity techniques and the cooperative algorithms to overcome the performance degradation.

Low-complexity cooperative relaying schemes based on the combination of the delay diversity (DD) technique and the MRC (maximal ratio combiner) receive diversity algorithm are proposed for a DVBSH-compliant hybrid satellite/terrestrial network (satellite to handheld - type A (SH-A) Architecture) [10], [11]. Both pros and cons of the additional frequency diversity afforded by the DD method are analyzed and the adoption of a simple algorithm for channel estimation is investigated [12] as a solution to exploit the performance improvement of the coded orthogonal frequency division multiplexing (OFDM) system.

In a hybrid satellite terrestrial relaying scheme, independent faded and delayed copies of the transmitted signal are received either from the satellite or from the relays; in particular, in order to implement a more realistic system, at the receiver end the signals coming from the relays are characterized by a higher power with respect to the ones coming from the satellite (not equal power-neq).

In the SALICE context the relays can be represented by gap fillers, identified with temporary mobile stations placed at the perimeter of the disaster site (MMNs). Moreover, the satellite-destination and the satellite-relay links are supposed to be alternating LOS/NLOS (line of sight/nonline of sight) visibility conditions, while a multipath channel (with Rayleigh distribution) is considered between the relays and the destination. It is important to note that the equivalent channel perceived by the end user is a hybrid channel, which consists of terrestrial and satellite components and is characterized by a mix of the LOS and the NLOS propagation conditions. 
Simulations results show that combining the cooperative DD technique with the MRC receive diversity scheme permits us to achieve interesting bit error rate (BER) performance that overcomes the performance of a terrestrial system through the exploitation of both the channel propagation features and the spatial diversity gain. The BER performance of two-relay systems in the city setting, assuming a double-antenna equipped receiver, is reported in figure 2; in particular the comparison between the cooperative DD scheme with and without the MRC receive diversity algorithm is depicted, showing the two-relay delay diversity - 2 receiving antennas (DD-2RX) system gain of 5-6 dB for $\mathrm{BER}=1 \mathrm{e}-04$ over the two-relay DD one.

It is worth highlighting that a two-element antennaequipped destination node, required for the implementation of the MRC algorithm, can be represented by an anchor node able to coordinate the rescuers of its team according to the information received from the ECC. With these assumptions the presence of two antennas at the receiver end does not represent a limitation in terms of equipment cost and size. In fact, thanks to the performance achieved with this diversity scheme, just one anchor node is required for a team of rescuers.

2) HAP /MANET cooperation: the strong heterogeneity of SALICE architecture poses several challenges in the definition of an efficient radio resource management (RRM) scheme. The employment of multimedia broadcast/multicast services (MBMS) in the incident area can enhance system performance, since multicast emergency transmissions can be delivered to groups of FRs at the same time; thus avoiding data duplications both in the core network and the over-the-air interface [13]. Further improvements in terms of system capacity can be achieved by extending cellular system coverage through the aid of self-organizing MANETs. Simulation campaigns have been carried out taking into account the altitude of the HAPs and the radius of the cells equal to 22 and $2.6 \mathrm{~km}$, respectively.

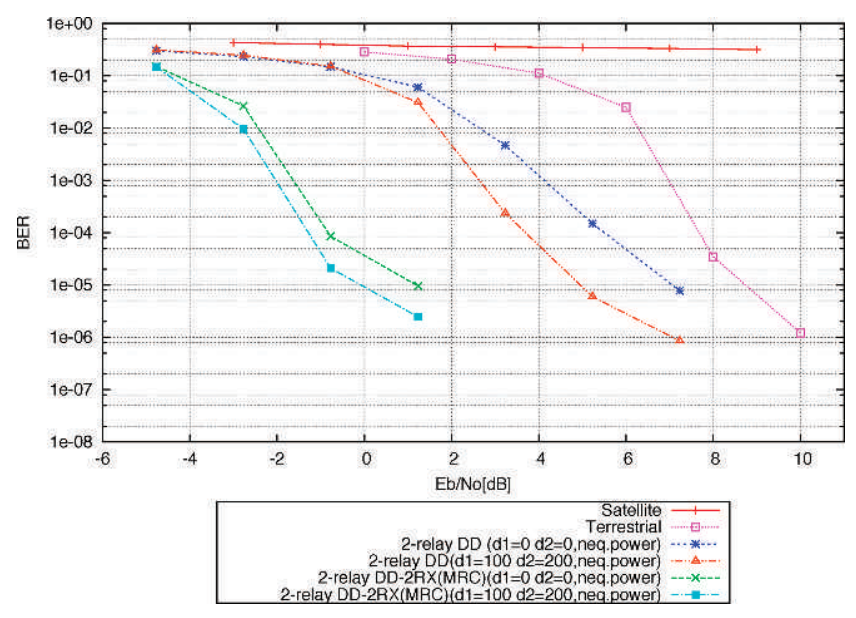

Figure 2.

Performance comparison of DVB-SH (SH-A Architecture: Mode 1K, Quadrature Phase Shift Keying (QPSK) system among: satellite only, terrestrial only, N-Relay Cooperative DD, and NRelay Cooperative DD combined with MRC, with $N=2$ in city environment.
Moreover, as for the HAP model, the channel is HighSpeed Downlink Shared Channel (HS-DSCH) and the path loss model is free space affected by rooftop scattering and multiple screen diffraction; on the other hand, the MANET propagation model and routing protocol are two ray ground and On-Demand Multicast Routing Protocol (ODMRP), respectively. Finally, the following application scenarios have been taken into account: (i) the terrestrial network involved in the disaster area is not available anymore and, in this case, long-range communications are guaranteed by a stand-alone HAP [14]; (ii) an FRs team is interested in receiving multicast emergency transmissions (e.g., download of images or maps) provided by ECC; (iii) HAP supports high-speed packet access (HSPA) connections; (iv) some of the end-devices of FRs (i.e., SALICE terminals) have both the HSPA and Wi-Fi radio interfaces and can act as anchor nodes (ANs).

Cooperation is performed through the AN, which downloads data traffic over an HSPA link and exchanges data with other FRs in the MANET over the Wi-Fi interface, thanks to the ODMRP. It is a mesh-based routing protocol for MANET that guarantees high scalability and reliability [15]. The most evident result obtained is the relevant incremental increase of system capacity due to the introduction of the cooperative multihop access, which depends on the specific scenario and on the reciprocal visibility of FRs devices. This increment does not require any augmentation of the cellular network infrastructure, because the access to the Wi-Fi network is directly offered by the terminal. From figure 3, an improvement in terms of packet delivery ratio (PDR) can be seen when the number of nodes connected to the same AN increases. Such an improvement is due to the mesh routing protocol supported by ODMRP algorithm that also allows an increment of the overall system capacity and scalability. The price to pay is in terms of delay (see figure 4) and data-rate reduction of the multicast users connected through the Wi-Fi interface. This is due to a higher packets collision when the number of packets per second provided

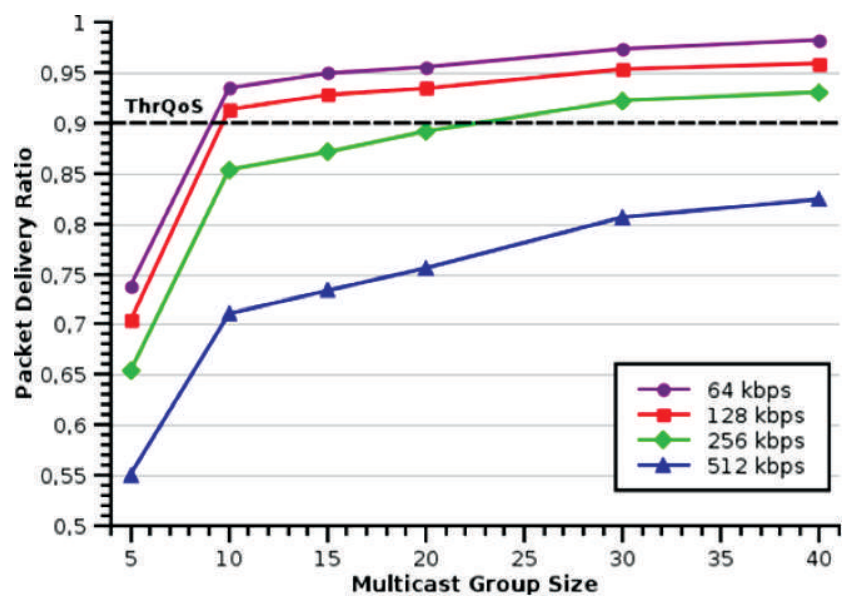

Figure 3.

PDR per multicast group as a function of the number of multicast receivers and HSPA data rate. 


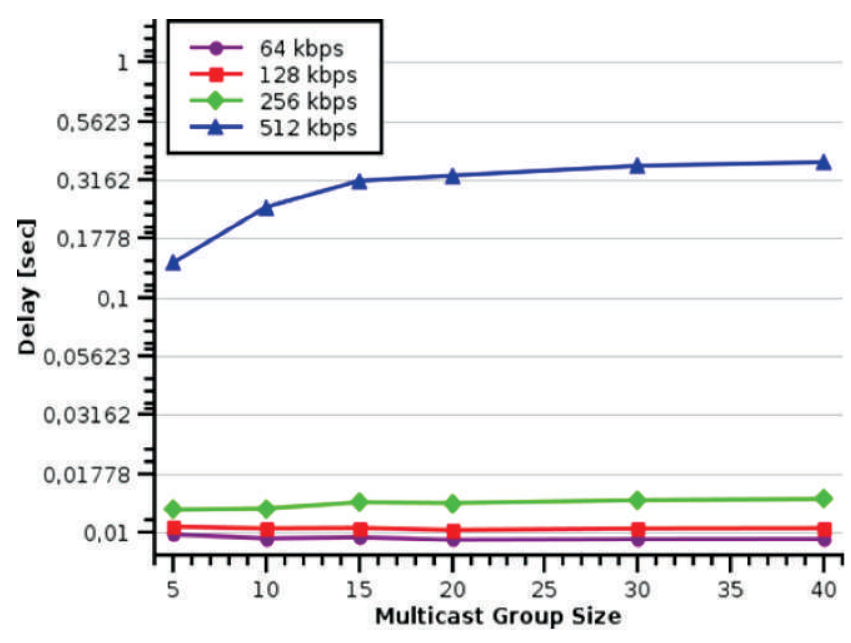

Figure 4.

Delay per multicast group as a function of the number of multicast receivers and HSPA data rate.

by a single AN increases. The dashed line represents a possible threshold (ThrQoS) of acceptable PDR. This threshold determines the maximum data rate supported by MANET, and, moreover, it defines the number of user equipment under which a HAP/MANET cooperation is not suitable.

In order to support cooperation and efficiently manage the HAP radio resources, the HSPA signaling feedback procedure based on the channel quality indicator (CQI) [16] can be modified foreseeing the introduction of further two fields: (i) the number of users belonging to the multicast group and (ii) the maximum data rate guaranteed by MANET for a given PDR. Modulation, coding scheme, and schedule policies will be adjusted every CQI report cycle [16] on the basis of feedback provided by the ANs with the purpose of guaranteeing a given PDR. The introduction of these new features into the HAP RRM requires negligible modifications in the ODMRP (on-demand multicast routing protocol). Specifically, new signaling and control packets have to be introduced to efficiently manage the multicast MANET receivers and to establish the maximum number of users connected to the AN. If cooperation is not feasible, HAP will activate point-to-point connections equal to the number of users requiring the multicast service, according to HSPA standard. Obviously, this situation leads to a significant reduction of system capacity.

3) Coexisting air interfaces in the IAN: an IAN should be used for both voice/video/data transmissions and wireless sensor communications in the local and/or personal area. Therefore, the definition of an IAN for emergency service management is described in the following. The user, e.g., a rescue team member, is provided with a relief member unit (RMU) which is a network node with multistandard capabilities and bridge functionalities. The wireless networks which are essential for the use of the RMU are a medium-range broadband wireless network (e.g., based on IEEE802.11) and a short-range low data rate (LDR) wireless sensor network (e.g., based on the standard IEEE802.15.4).
The medium-range broadband wireless network is used for communication between users in ad hoc mode permitting the exchange of videos and high quality images. The short-range LDR wireless sensor network is composed of a set of sensors for location and context detection (e.g. vital sign sensor, temperature sensor, GNSS receiver, indoor positioning devices) with an energy-efficient air interface. The physical layer (PHY) transmission bit rate of the LDR interface ranges from few bps to $250 \mathrm{kbps}$.

The IAN should allow processing and fusion of location/ context data collected by sensors and data delivery between network nodes and the access network. Since most of the wireless sensor networks and medium-range broadband wireless networks exploit unlicensed frequency bands and they can partially or totally overlap in frequency, coexistence issues have to be carefully addressed.

An important aspect that has been investigated in the project is the energy efficiency of the wireless sensor network when running in proximity to a medium-range broadband wireless network. Experimental trials have been carried out using a Wi-Fi node and a short-range wireless transceiver CC2500/2550DK with a development kit developed by Chipcon. The energy efficiency of the short-range wireless link has been computed as follows:

$$
\eta=N(1-p) / E_{t o t}
$$

where $N$ is total number of bit per packet, $p$ is the packet error rate, and Etot is the total energy consumed by the transceiver for the transmission of one packet.

In order to characterize the behavior of the coexisting air interfaces in the IAN, the energy efficiency was used as the performance metric and it has been evaluated as a function of the received signal strength indicator (RSSI). Figures 5 and 6 show the experimental results of the energy efficiency of the LDR short-range wireless link with and without interference. As a first result, it is worth noting that the energy efficiency is maximized by a given value of the RSSI. This is an experimental confirmation of the result analytically presented in [17] and [18], where the signal to noise ratio (SNR) was used instead of the RSSI. As can be noticed from the comparison of figures 5 and 6 , the maximum value of the energy efficiency is almost one order of magnitude lower when an interfering Wi-Fi node is present. Furthermore, the RSSI, which is required to maximize the energy efficiency, is $18 \mathrm{dBm}$ higher. Therefore, the performance of the short-range link is degraded by the interfering Wi-Fi node and, as proposed in [19], the use of a coexistence algorithm is of paramount importance.

\section{ANALYSIS AND DEVELOPMENT OF A RECDNFIGURABLE AND COOPERATIVE SYSTEM}

The organization, the timely support, and the safety of the FR teams rely on the availability of a robust communica- 


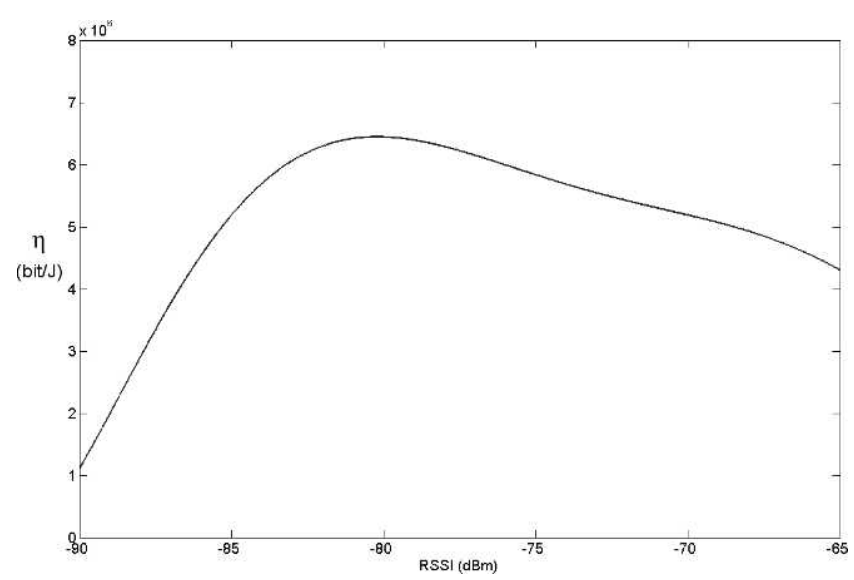

Figure 5.

Energy efficiency of the LDR link versus the RSSI without interference.

tions system and on the localization information. Indeed, knowing timely and accurately the position of the rescue entities (persons and means) is a fundamental enhancement in the coordination and planning of emergency and disaster relief operations, both in terms of the efficacy and safety of the intervention. Moreover, the restoration of the wireless connectivity that has been interrupted by the partial or total crash of the existing network infrastructure represents a relevant communication functionality that should be ensured in the incident area network. To address these two main issues, the study and development of an integrated reconfigurable SDR device able to support the required NAV/COM services for the emergency management and the definition of the possible countermeasures to the evolving emergency scenario were identified as the main objectives of this research activity.

In the following, the developed integrated SDR NAV/ COM demonstrator is described together with the implemented cooperative localization solution and the analyzed multimodal SDR-based solution.

1) Cooperative localization approach: integrated SDR NAV/COM terminal: Satellite-based systems, for both positioning and communications, are unavoidable in situations of public emergency, where terrestrial infrastructures could be severely damaged due to disruptive events. Worldwide satellite localization is offered by Global Navigation Satellite Systems (GNSS), namely GPS, GLONASS, and, in the future, Galileo, enhanced by various kinds of augmentation services (e.g., European Geostationary Navigation Overlay Service (EGNOS), Wide Area Augmentation System (WASS)) [20]; however, these systems suffer from the weakness (or even the blockage) of their signals when the receiver operates in nonopen sky (nonline of sight) conditions with respect to the satellites. This limitation is critical for emergency interventions, which require highly reliable positioning systems in terms of availability and continuity. Assisted GPS (AGPS) is offered today on most commercial general-purpose GPS chips.

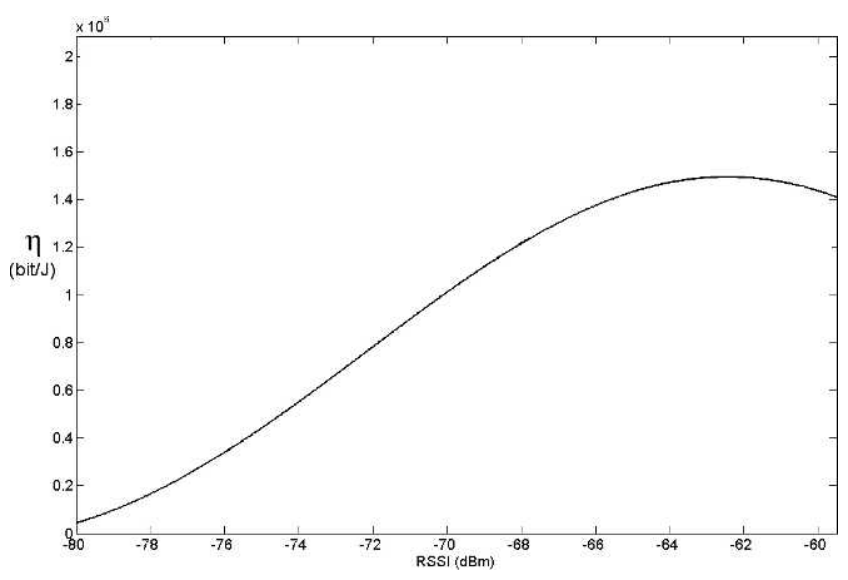

Figure 6.

Energy efficiency of the LDR link versus the RSSI with an interferent Wi-Fi transmitter.

Assistance information transmitted to the receiver can notably reduce the time to first fix (TTFF) and improve acquisition sensitivity [21]. Typical assistance data are: reference time, ephemeris, almanac, approximate position of the user, Doppler frequency, and code delay. However, AGPS is based on the cellular network and therefore cannot be assumed sufficiently reliable in public emergency situations. Nonetheless, AGPS can represent the basis for an ad hoc framework aimed at providing assistance (aiding) for the localization of a receiver in light-indoor conditions (i.e., for which the satellite signals are seen with a low carrier to noise ratio $(\mathrm{C} / \mathrm{NO})$ ratio but not as completely blocked). This framework, named peers-based aiding to GNSS [22], [23], is intended for a scenario in which "peer terminals" assist each other in their positioning task. The fundamental hypothesis of peers-based aiding to GNSS is that some aiding terminals have open sky visibility to the satellites, while other aided terminals, relatively close to the first ones, need their assistance to acquire the satellite signals. The aiding data are exchanged via an onboard communication module able to establish reliable short/medium-range point-to-point radio links in a partially connected network.

As the most difficult task faced by a terminal in light indoor is the acquisition of the satellite signals, possibly with a very low $\mathrm{C} / \mathrm{N} 0$ ratio, the main aid, which is provided by an open-sky terminal, is in the acquisition phase. Conceptually, the aiding terminal informs the aided one about the Doppler frequency for each satellite in view, as well as the position of the data bit transition. Thanks to the relative closeness of the two peers with respect to the satellites, these estimates can be used to reduce the dimension of the search space along the time and frequency space. Furthermore, the information about the position of the data bit transition allows setting an adequate number of long coherent and noncoherent integrations that enhance the signal-to-noise ratio at the acquisition stage. However, the effective exploitation of such aids is possible only under the hypothesis of a fine synchronization between the 
internal clocks of the peers. A synchronization between two peer clocks with less than $1 \mu$ s accuracy (GPS coarse acquisition (C/A) chip width) is required for the efficacy of the peers-based aided acquisition. However this limit cannot be reached with ordinary communication cards and common PC internal clocks without a dedicated hardware and suitable network synchronization protocols. For this reason an ad hoc procedure to synchronize the clock offset of two terminals with poor clock performance has been designed and positively tested [22]. The two terminals must simultaneously receive the signal from at least one satellite. This is not uncommon in light indoor conditions, when at least one satellite has visibility enough to allow the tracking of its signal in the GPS receiver, while all the others are too attenuated. The synchronization phase aims at aligning the clocks of two terminals by estimating their respective offsets. It has been demonstrated that the same procedure can also provide an estimate of the frequency offset between the local oscillators of the GPS front ends installed on the two terminals [22]. These two parameters (clock offset and frequency offset) can be used then as aiding data in the cooperative localization procedure.

Once the two peers are synchronized, the cooperative peers-based acquisition is enabled [23]. Differently from AGPS, a peers-based approach exploits as much as possible the mutual proximity of the terminals involved in the aiding procedure, making the reasonable assumption that the local environment seen by a certain terminal is similar enough to that seen by close terminals, and allows the beneficial exchange of "local" information such as C/N0 ratios, Doppler frequency shifts, bit synchronization, receiver altitude, and so on [24]-[26]. The cooperative localization techniques that have been studied have been also implemented and demonstrated in a software defined radio NAV/COM device (the SALICE terminal), which enables the localization of FRs also in partially blocked satellite environment. The SALICE terminal is an integrated NAV/COM device that consists of two coexisting and cooperating software modules: the navigation software (SW) module, which allows the localization of the terminal itself, and the communication SW module, which deals with the transmission of position parameters.

The two modules, which allow the implementation of the cooperative localization algorithm, are separated programs running on the same on-the-shelf laptop PC that has been used for the test-bed. Particularly, the navigation module is a controlled TCP/IP stack protocol that is based on the NGene/N-Grab platform [22], while the communication module is based on the GnuRadio [23]. The overall structure of the SALICE terminal is shown in figure 7.

In order to validate the proposed cooperative NAV/ COM system a test-bed composed of two SALICE terminals (representing two different users belonging to the same cluster of FR) has been set up.

The final validation and performance evaluation test aimed at verifying the results that can be achieved in a real light-indoor scenario; moreover, specific goals can be

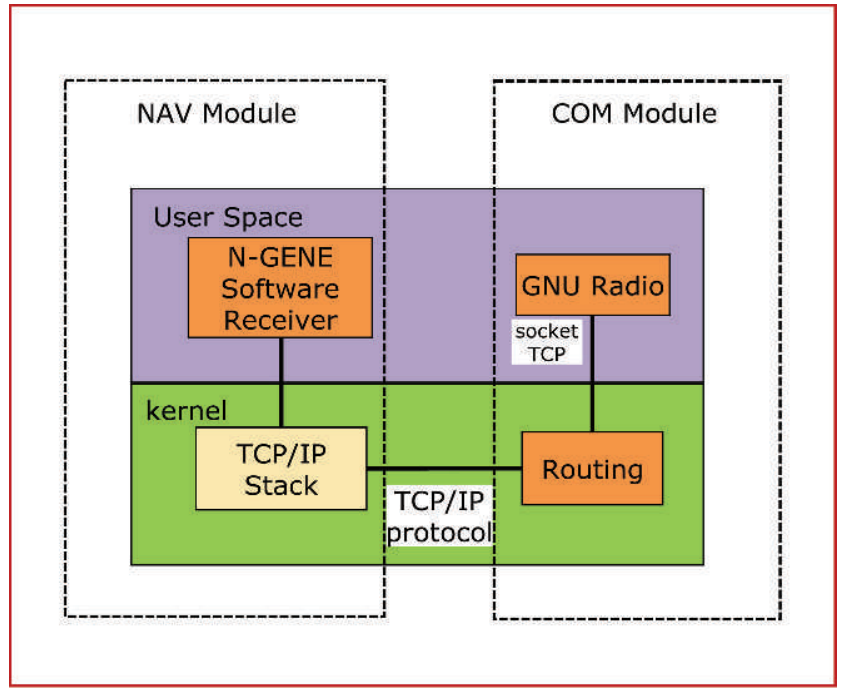

Figure 7.

Integration of NAV/COM Systems in SALICE terminal.

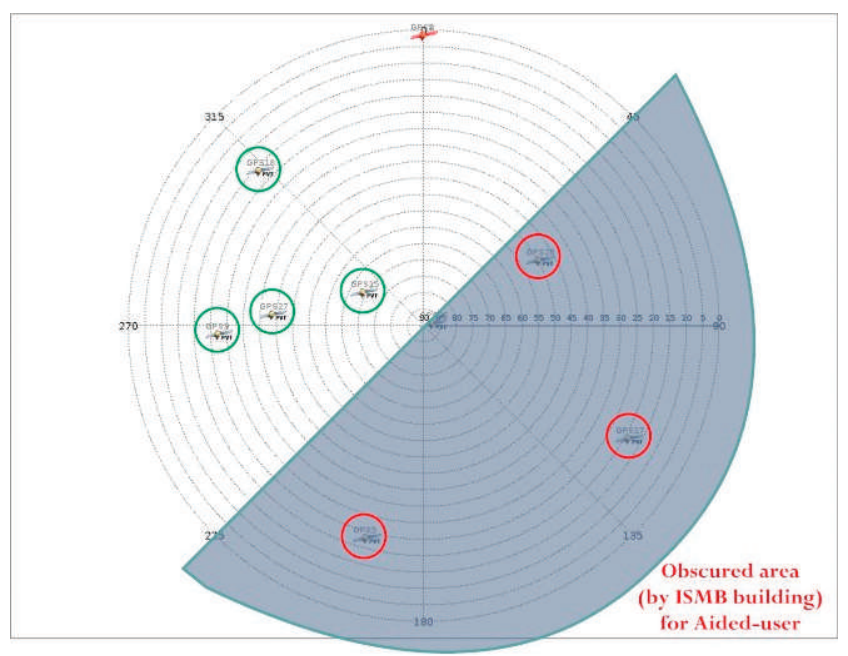

Figure 8.

Position of GPS satellites for SALICE terminal 2.

identified in the verification of the GNSS aiding algorithm and in its integration with SDR COM modules. The test included the communication link establishment, the synchronization issue, the aiding data creation, and the exploitation of the aid in the acquisition step. In particular, the SALICE terminal 1 is in open-sky condition and has full visibility of GPS satellites, while the terminal 2 is in a light-indoor position. Nonetheless, this terminal can still find some GPS satellite in LOS visibility but not enough to perform a correct localization independently. Therefore, the SALICE terminal 1 (in full visibility) plays the role of the aiding user for the SALICE terminal 2 (in partially blocked visibility), which becomes the aided user. A time synchronization between the two SALICE terminals is performed, thanks to the Network Time Protocol (NTP) 
protocol and the IP tunnel which has been created through the GnuRadio. The distance between the two SALICE terminals is on the order of few meters.

In figure 8 the position of GPS satellites for the SALICE terminal 2 is depicted. The GPS satellites identified by the red circles have not been considered in the aiding algorithm. If at least one of the satellites identified by green circles is in visibility, the aiding algorithm can be performed and, possibly, the terminal 2 can be aided by the aiding user (terminal 1).

After a proper time offset compensation, the synchronization can be accomplished and the algorithm can be applied at every satellite positioned in the visible zone of the aided user. The aiding algorithm reduces the probability of false detection by generating a higher correlation peak with respect to the acquisition without aid and to lower the TTFF thanks to a reduction of the search space to be evaluated.

As an example, the results achieved by the aiding algorithm for the satellites that were positioned in the visible zone of the aided user during the test, namely PRN9, PRN18, and PRN27, are reported. The sampling frequency and the coherent integration time are assumed equal to $16.3676 \mathrm{MHz}$ and $20 \mathrm{~ms}$, respectively.

In figure 9 the position of GPS satellite PRN9 with respect to the antenna of the aided terminal is highlighted (figure 9a). Moreover, the search spaces for the satellite are shown both in the cases of aided and not aided acquisition. Particularly, the search space (frequency offset and code delay are indicated on the axes) is characterized by the absence of correlation peak when no aiding procedure is adopted (figure $9 b)$. On the contrary, when the aided data are used in the acquisition procedure, a clearly visible peak is obtained as can be seen from figure $9 \mathrm{~d}$. Besides, the dimension of the search space intervals to be evaluated are remarkably shorter; therefore, we end up achieving an improvement on terms of TTFF. Finally, the correlation peak with respect to frequency value is depicted in figure $9 \mathrm{c}$. The same situation can be verified for satellite 18 and 27, as indicated in figures 10 and 11, which otherwise would not be acquired because of their low carrier-to-noise ratio. In both cases, a high peak is achieved together with a low noise value. Hence, the probability of false detection is reduced.
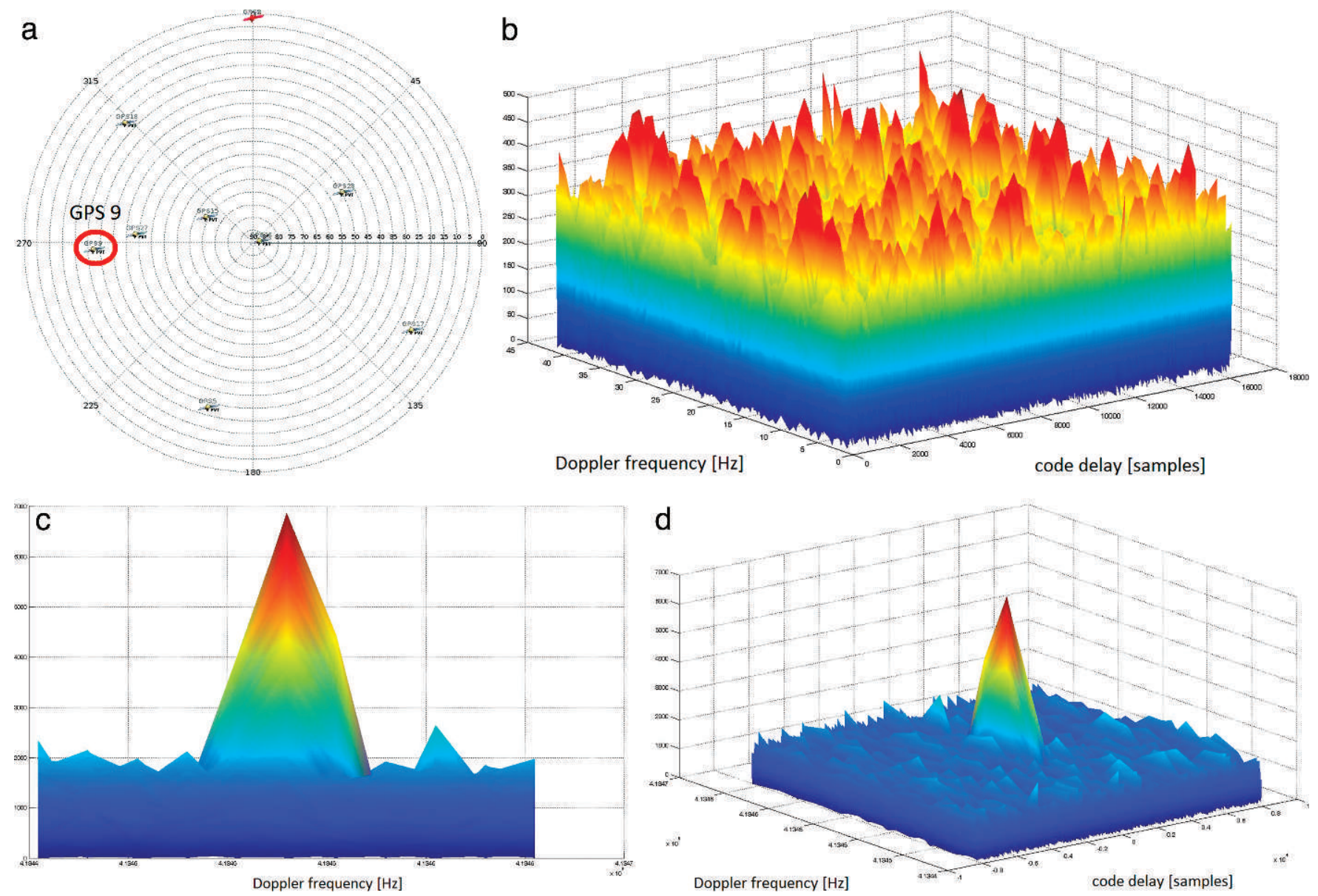

Figure 9.

Comparison between the search spaces of satellite PRN9 (without and with the aiding procedure). (a) GPS-PRN9 Satellite position. (b) search space (2-D), GPS-PRN9, no aiding, (c) search space, GPS-PRN9, aided procedure, (d) search space (2-D), GPS-PRN9, aided procedure. 

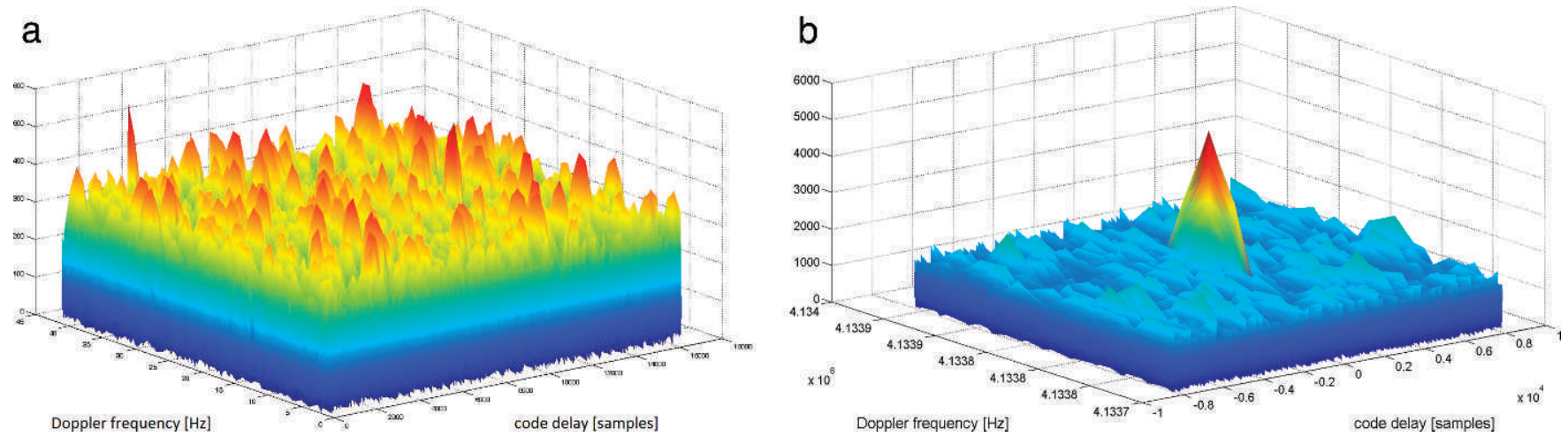

\section{Figure 10.}

Comparison between the search spaces of satellite PRN18 (without and with the aiding procedure). (a) search space (2-D), GPS-PRN18, no aiding, (b) search space (2-D), GPS-PRN18, aided procedure.
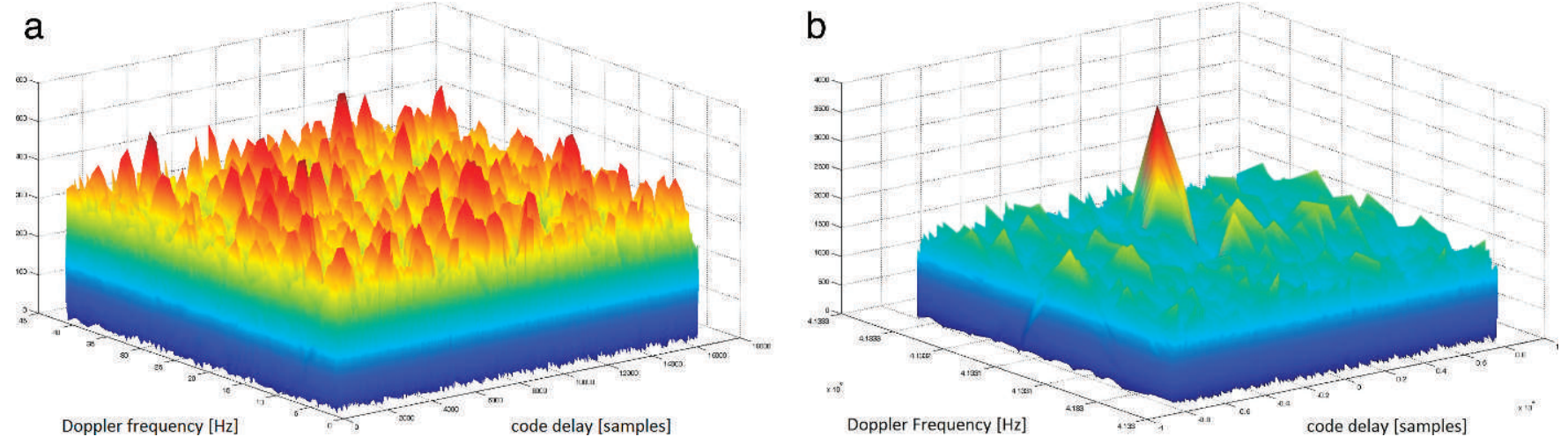

Figure 11.

Comparison between the search spaces of satellite PRN27 (without and with the aiding procedure). (a) search space (2-D), GPS-PRN27, no aiding. (b) search space (2-D), GPS-PRN27, aided procedure.

To conclude, the proposed aided acquisition procedure affords the following objectives:

- Improvement of receiver sensitivity

- Reduction of probability of false detection

- TTFF improvement

2) Reconfigurability: SDR-based multimodal terminal: The problem of restoring the lost wireless connectivity in the incident area network has been already considered by the EU Wireless Infrastructure over Satellite for Emergency Communication (WISWCOM) project [27], [28], and the envisaged solution consists of a transportable multistandard terminal obtained by integrating in a single box a pico-cellular base station for GSM, another one for UMTS, a Wi-Fi access point, and, finally, a satellite transceiver used to extend the coverage. We think that a step ahead with respect to state of the art should be done by exploiting the capabilities and the potentialities of software-defined radio (SDR) technologies. In this framework, SDR should provide increased flexibility and reconfigurability, together with other benefits in terms of reduced size and weight of the terminal, reduced power consumption, etc. Moreover, SDR may allow the shift to an opportunistic perspective, where the reconfigurable multimodal terminal seeks exploitable radio resources in free unlicensed bandwidth or in bandwidth portions previously exploited by cellular services that are hindered to work. During SALICE project activities, the feasibility of multimodal terminal reconfigurability has been studied and emulated by means of a GnuRadio test-bed using an universal software radio peripheral hardware (USRP HW) board from Ettus Corporation. Different scenarios have been considered in the emulations, i.e., indoor and outdoor transmission, LOS and NLOS situations, etc. In particular, the transmission frequency was in the 2.4 GHz and following standard PHY-layer signal formats were considered:

- GSM signal (single carrier, GMSK modulation, channel bit rate: $271 \mathrm{~Kb} / \mathrm{s}$ )

- IEEE 802.11g OFDM mode ( $N=64$ subcarriers, binary phase shift keying (BPSK), QPSK, quadrature amplitude modulation (QAM), and 64-QAM modulations)

- WiMax OFDM signal ( $N$ = 256 subcarriers, QPSK, 16QAM and 64-QAM modulations)

In order to cope with bandwidth constraints of a USRP device, the multiplexing of the signals was done using a time 
division multiplex (TDM) strategy transmitting at a data rate, which was conveniently scaled (see [21], [23] for further details). The reconfigurable baseband receiver was executed on a laptop supplied with a dual-core CELERON processor T3100 with a CPU clock at $1.90 \mathrm{GHz}$ and 2.9 GB of RAM. The operating system was the Linux Ubuntu release 9.04. We first verified the capability of the receiver to recognize the presence (or the absence) of a transmission mode in a given time slot and to reconfigure itself on the basis of the detected transmission mode. Such a capability has been fully verified by means of intensive testing trials. The mode identification became impossible only at a long distance (about $50 \mathrm{~m}$ ). Among the experimental results, we can show the values of the normalized power received in each time slot, where the presence of a transmission mode has been detected. In figure 12 , such values are plotted for two LOS scenarios. The first is characterized by a strong LOS signal component; the second is characterized by a weak LOS component. The average signal-to-noise ratio reduction and the power fluctuations are evident at a glance for the weak LOS case, as well as the increase of measured BER.

\section{SALICE DISTINCTIVE FEATURES}

The SALICE project focused on:

- The integration of different technologies through the adoption and development of transmission and resource management techniques and algorithms suitable for a quick deployment of an emergency network able to provide the connectivity in the first hours after the disaster

- The integration of navigation and communication functionalities through the SDR technology in a single device able to guarantee navigation information also in critical scenarios as the ones characterized by a partial satellite visibility

Therefore, differently from some of the main emergency systems proposed by European projects (see table 1), which are limited to the study of a specific emergency situation need, the simultaneous investigation of the previously described aspects has permitted defining a whole emergency system that fulfils the main requirements of emergency situation management: the coverage of the incident area with connectivity with the external world and the integration of the communication and navigation capabilities in order to cope with difficult operational conditions. It is worth highlighting that the purpose of table 1 is to show which of the main functionalities achieved by the SALICE system were also considered in other projects, emphasizing the SALICE comprehensive emergency system and its innovative features.

\section{CDNELUSIONS}

In order to meet the telecommunication requirements for the management of the first-response phase of an emergency situation, SALICE project activities have been focused on two main research areas: achieving global coverage of the emergency area and providing the rescuers with advanced NAV /COM capable devices.

The identification of the SALICE baseline scenario, the definition of the emergency system requirements, and the definition of the architectural specifications have permitted us to investigate both the long- and shortrange technologies able to guarantee the required global coverage (within the area of intervention and between the emergency area and the external areas). The integration and cooperation of space and terrestrial segments was investigated together with the analysis of the PHY technologies, interference effects, and protocol solutions, which can be effectively adopted in the heterogeneous IAN for location/environment data delivery.
Figure 12.

Normalized power received in the three time division multiple access (TDMA) slots for the different standard signals: outdoor scenarios with weak LOS (green line) and strong LOS component (red line). 
Table 1.

SALICE Functionalities Comparison Matrix

\begin{tabular}{|l|c|c|c|}
\hline SALICE Investigated System Features & WISECOM [28] & CHORIST [29] & PEACE [30] \\
\hline $\begin{array}{l}\text { Terrestrial Communication Technologies } \\
\text { Integration }\end{array}$ & $\mathrm{x}$ & $\mathrm{x}$ & $\mathrm{x}$ \\
\hline Satellite and Terrestria/ Technologies Integration & $\mathrm{x}$ & $\mathrm{x}$ \\
\hline HAP and Terrestrial Technologies Integration & $\mathrm{x}$ & \\
\hline $\begin{array}{l}\text { Terrestrial Communication Systems for } \\
\text { Localization Purpose }\end{array}$ & $\mathrm{x}$ & \\
\hline $\begin{array}{l}\text { Navigation and Communication } \\
\text { Capabilities Integration }\end{array}$ & & \\
\hline Context Awareness and NAV/COM SDR Device & & \\
\hline
\end{tabular}

The viability of an SDR approach in the design of an intelligent NAV/COM device has been thoroughly studied. For the implementation of a reconfigurable and flexible user terminal able to modify itself to cope with any NAV/COM requirements, the integration in a single box of several communication standards and of communication and localization/navigation systems has been considered and the main achieved results have been presented: an SDR NAV/COM demonstrator for rescue operations management in an emergency scenario and a multimodal reconfigurable terminal.

As confirmed by the obtained results, the solutions proposed to cope with the main telecommunication problems of an emergency situation management represent promising strategies that can be seen as a starting point for further investigation.

On a more general point, the SALICE project has proven that space and terrestrial communication networks and navigation and communication systems must complement each other in emergency scenarios. In fact, where terrestrial networks are partially or totally destroyed by the disaster, spacebased systems can effectively complement surviving telecommunication infrastructure (e.g., still operating cellular and wireless systems), MANETs, and WSNs (wireless sensor networks), to provide communication restoration with wide coverage and efficient support to first responders' interventions. Space-based systems also may offer back-hauling capability to self-organizing distributed wireless networks and help to decongest the (surviving) wireless terrestrial infrastructure when any. On the other hand, the cooperation between navigation and communication services allows the assistance of all the FRs in their operations when bad propagation conditions are experienced. Future studies and development could include a monitoring system as complementary to the communication and navigation systems.

\section{REFERENCES}

[1] Berioli, M., Mulero Chaves, J., Courville, N., Boutry, P., Fondere, J.-L., Skinnemoen, H., et al. Wisecom: A rapidly deployable satellite backhauling system for emergency situations. International Journal of Satellite Communications and Networking, Vol. 29, 5 (2011), 419-440.

[2] Berioli, M., Molinaro, A., Morosi, S., and Scalise, S. Aerospace communications for emergency applications. Proceedings of IEEE, Vol. 99, 11 (2011), 1922-1938.

[3] Ahn, D., Kim, H., Ahn, J., and Park, D.-C. Integrated/hybrid satellite and terrestrial networks for satellite imt-advanced services. International Journal of Satellite Communications and Networking, Vol. 29, 3 (2011), 269-282.

[4] Del Re, E. and Ruggieri, M. Satellite Communications and Navigation Systems. Springer, 2007.

[5] Paillassa, B., Escrig, B., Dhaou, R., Boucheret, M., and Bes, C. Improving satellite services with cooperative communications. International Journal of Satellite Communications and Networking, Vol. 29, 6 (2011), 479-500.

[6] ETSI. Requirements for communication between authorities/ organizations during emergencies (EMTEL). ETSI Recommendation, TS 102181 V.1.1., ETSI Std., 2005.

[7] Jebril, A., Scucchia, L., Lucente, M., Ruggieri, M., Cambriani, P., Rossi, T., et al. The wave mission payload. In Proceedings of 2005 IEEE Aerospace Conference, Big Sky, MT, March 5-12, 2005.

[8] Araniti, G., De Sanctis, M., Spinella, S. C., Monti, M., Cianca, E., Molinaro, A., et al. Cooperative terminals for incident area networks. In First International Conference on Wireless VITAE 2009, Aalborg, Denmark, May, 2009.

[9] Araniti, G., De Sanctis, M., Spinella, S. C., Monti, M., Cianca, E., Molinaro, A., et al., Hybrid system hap-wifi for incident area network. In 2nd International Conference on Personal Satellite Services PSATS'10, Rome, Italy, February, 2010.

[10] System Specifications for Satellite Services to Handheld Devices (SH) Below $3 \mathrm{GHz}$ European, Digital Video Broadcasting (DVB) Std. ETSI TS 102585 V1.1.1, 2007.

[11] DVB-SH Implementation Guidelines, Digital Video Broadcasting (DVB) Std., DVB Document A120. ETSI TS 102584 V1.1.1 (2008-12), 2008.

[12] Morosi, S., Jayousi, S., and Del Re, E. Cooperative delay diversity scheme with low complexity channel estimation in inte- 
grated satellite/terrestrial systems. In Wireless Conference (EW), 2010 European, 2010, 496-502.

[13] Multimedia Broadcast/Multicast Service (MBMS); Architecture and Functional Description (Release 6). 3GPP Std., 3GPP TS 23.286 v6.0.0 (2003-09), 2003.

[14] Araniti, G., Iera, A., and Molinaro, A. The role of haps in supporting multimedia broadcast and multicast services in terrestrial-satellite integrated systems. Wireless Personal Communications, 2005.

[15] Obraczka, G. T. K. and Viswanath, K. Exploring mesh- and treebased multicast routing protocols for manets, IEEE Transactions on Mobile Computing, Vol. 5, 1 (2006), 28-42.

[16] Holma, A. T. H. HSDPA/HSUPA for UMTS High Speed Radio Access for Mobile Communications. John Wiley and Sons, 2006.

[17] De Sanctis, M., Cianca, E., and Ruggieri, M. Energy efficient transmit power control for hdr wpan. In 17th Annual IEEE International Symposium on Personal, Indoor and Mobile Radio Communications (PIMRC'06), Helsinki, Finland, Sept. 2006.

[18] De Sanctis, M., Cianca, E., and Joshi, V. Energy efficient wireless networks towards green communications, Wireless Personal Communications, an International Journal, Kluwer, vol. 59, no. 3, pp. 537-552, August 2011

[19] De Sanctis, M., Monti, M., Ruggieri, M., and Prasad, R. A collaborative coexistence mechanism for ieee 802.15.3 and 802.15.4 wpans. In Proceedings of 20th Annual IEEE International Symposium on Personal, Indoor and Mobile Radio Communications (PIMRC 09), Tokyo, Japan, Sept. 2009.

[20] Fernandez-Prades, C., Lo Presti, L., and Falletti, E. Satellite radiolocalization from GPS to GNSS and beyond: Novel technologies and applications for civil mass market. Proceedings of the IEEE, Vol. 99, 11 (2011), 1882-1904.

[21] Van Diggelen, F. A-GPS: Assisted GPS, GNSS, and SBAS. Norwood, MA: Artech House, 2009.

[22] Digenti, S., Nicola, M., Lo Presti, L., and Pini, M. Technique for the estimation of PC clock offset in a GNSS-aided network of collaborative users. In Proceedings of the 5th ESA Workshop on Satellite Navigation Technologies (NAVITEC'10), Noordwijk, The Netherlands, Dec. 2010.

[23] Panizza, M., Sacchi, C., Varela-Miguez, J., Morosi, S., Vettori, L. Digenti, S., et al. Feasibility study of an SDR based reconfigurable terminal for emergency applications. In 2011 IEEE Aerospace Conference, Big Sky, MT, March 5-12, 2011.

[24] Lo Presti, L., Margaria, D., and Rao, M. Novel techniques for a cooperative positioning approach based on peer-to-peer networks. In Proceedings of the Int'l Conf. on Data Flow, From Space to Earth, Venezia, Italy, March 2011.

[25] Margaria, D., Lo Presti, L., Kassabian, N., and Samson, J. A new peer-to-peer aided acquisition approach exploiting C/N0 aiding. In Proceedings of the 5th ESA Workshop on Satellite Navigation Technologies (NAVITEC'10), Noordwijk, The Netherlands, Dec. 2010.

[26] Rao, M., Lo Presti, L., and Samson, J. Peer to peer equation augmentation for an altitude aided GNSS receiver. In Proceedings of the IEEE 72nd Vehicular Tech. Conf. Fall (VTC 2010-Fall), Ottawa, ON, Sept. 2010.

[27] Wisecom. http://www.wisecom-fp6.eu/

[28] Berioli, M., Courville, N., and W. M., Emergency communications over satellite: the wisecom approach. In Proc. of $16^{\text {th }}$ IST Mobile and Wireless Communications Summit, Budapest, Hungary, July 2007, $1-5$.

[29] Aiache, H., Knopp, R., Koufos, K., Salovuori, H., and S. P., Increasing public safety communications interoperability: The chorist broadband and wideband rapidly deployable systems. In Proceedings of IEEE Safety Comm Workshop, Dresden, Jun. 2009, 1-6.

[30] Politis, C., Dagiuklas,T., Rodriguez, J., and R. Y., Peace: Ip-based emergency applications and services for next generation networks. In Proceedings of International TEMU (Telecommunications and Multimedia) Conference, Crete, Jul. 2008. 